\title{
Telemedicine for palliative care: Current and future challenges
}

\begin{abstract}
Dear Editor,
We read with interest the article published in the June 2021 issue of the Annals titled "Use of telemedicine in healthcare during COVID-19 in Pakistan: Lessons, legislation challenges and future perspective". ${ }^{1}$ We would like to share our perspectives on using telemedicine to deliver palliative care during the COVID-19 pandemic.
\end{abstract}

On 23 January 2020, Singapore reported its first confirmed case of COVID-19 and between 7 April 2020 and 1 June 2020, a "circuit breaker" was implemented. During this period, non-essential medical services and procedures were deferred and encouraged to be delivered remotely if possible. ${ }^{2}$ At the same time, buildings were converted to care facilities, and professionals were redeployed away from their primary roles to meet the demands of curbing COVID-19 cases. Therefore, our palliative care team adjusted the delivery of its services to adapt to the changes brought about by the pandemic. ${ }^{3}$

Currently, Singapore is one of the countries in Asia with guidelines to regulate telemedicine, which has resulted in a thriving ecosystem of digital health providers in Singapore. Studies conducted across Europe have found that using telemedicine to provide palliative care improved patients' access to healthcare professionals from home, enhanced their sense of security and safety, and allowed a close connectedness with their healthcare providers. ${ }^{4}$ However, patients in Singapore may have different levels of technological and health literacy compared to Western patients. Furthermore, providing palliative care remotely is relatively new, and few studies have been conducted in Singapore to explore its acceptability. Therefore, our team designed a study to pilot the acceptability of providing palliative care via telemedicine to advanced cancer patients in Singapore.

This programme was overseen by a palliative care consultant (at 0.1 full-time equivalent [FTE]) and a palliative care nurse (at 0.6 FTE). Upon referral from oncologists, patients were screened for recruitment into the study. The telemedicine service provided as part of the study consisted of an initial video consult with a palliative care nurse and consultant, targeting patients' reported symptoms and problems (Week 1). For the subsequent 11 weeks (Week 2-12), patients were monitored weekly through the Integrated Palliative care Outcome Scale (IPOS), a brief 10-item tool that assesses psycho-emotional, practical and information needs, together with symptoms common to advanced cancer patients. Patients with mild, and moderate to severe problems identified on the IPOS were reviewed and managed by the palliative care nurse, within 1 and 3 working days, respective to the severity of problems. The palliative care nurse remained the primary point of contact via telemedicine. Multidisciplinary meetings between the palliative care consultant, nurse and oncologist were conducted as needed. At the end of the study (Week 13), patients were invited to complete an evaluation form regarding the acceptability of the service. The inclusion and exclusion criteria, and study procedures are presented in Fig. 1.

Of the 51 eligible patients introduced to the study by the palliative care nurse, 31 patients consented to participate between June 2020 and December 2020. The median age of patients who accepted the service was lower (66 years) than those who rejected the service (70 years). Willingness to accept the service was associated with patients' sex, marital status and paying class. Male patients, patients who are married, or of private paying class were more likely to accept the service.

Patients declined to participate in the study for several reasons, including preferring to receive in-person consultations $(50 \%)$, perceiving that they did not require palliative care service $(33.33 \%)$, finding the weekly questionnaires too troublesome to complete $(5.56 \%)$, having concerns about the cost of the service $(5.56 \%)$, and concerns over operating the videoconferencing application or online questionnaires $(5.56 \%)$. The weekly completion rate of the IPOS by participants ranged from $62.5 \%$ to $96.2 \%$, and the overall completion rate was $75.1 \%$.

As this service was primarily led by the palliative care nurse, and due to the frequent communication between the nurse and patients over the course of the study period, a strong rapport was fostered between them by the end of 12 weeks. However, this led to some patients and caregivers requesting for continued services beyond the study period, or patients continuing to contact the nurse for support even after their participation in the study had ended.

Feedback about the acceptability of our programme was generally positive. Most patients reported that our 


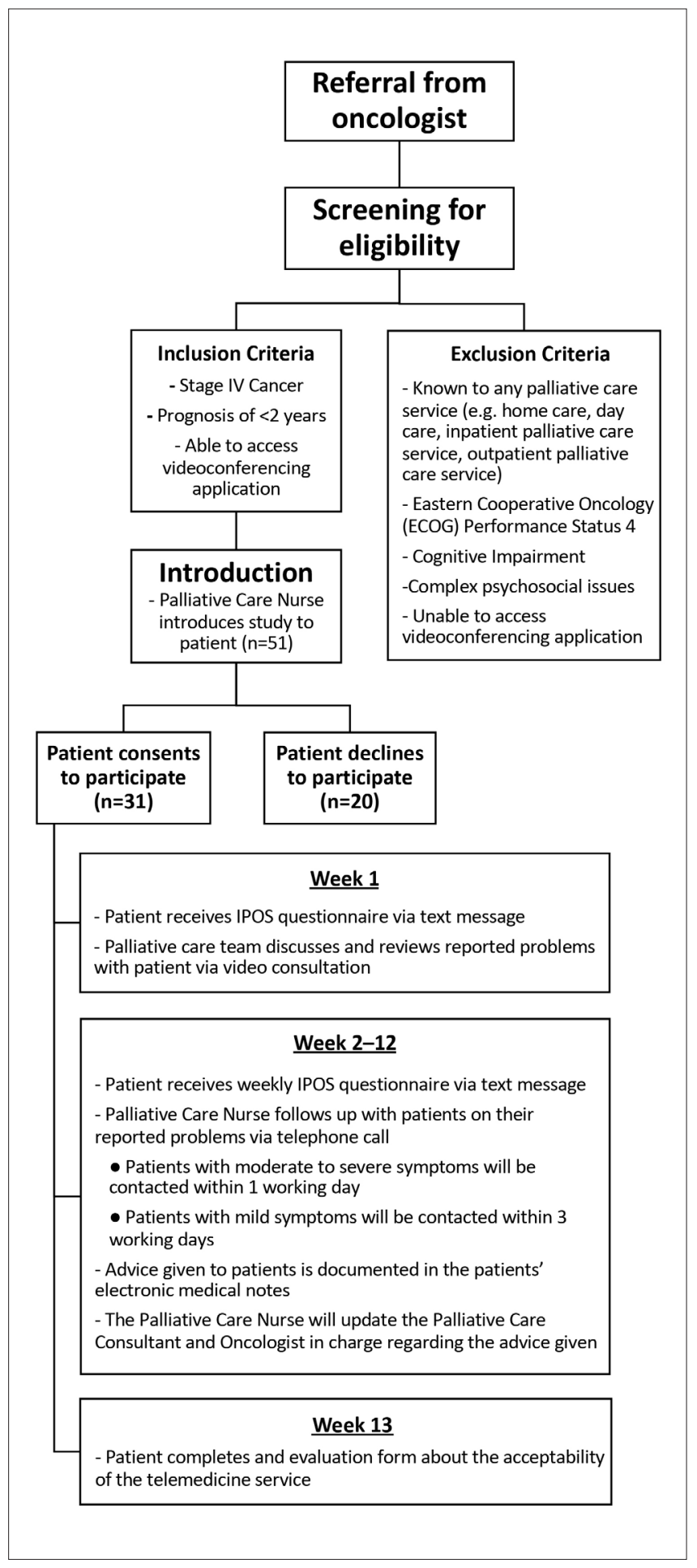

Fig. 1. Study procedure, and inclusion and exclusion criteria. IPOS: Integrated Palliative care Outcome Scale

programme had met most $(64.3 \%)$ or all of their needs $(28.6 \%)$, and had either somewhat helped them to deal more effectively with their problems $(50.0 \%)$ or helped them greatly $(50.0 \%)$. All of the patients also commented that they were satisfied with our programme and would return to it if they needed to seek help again. However, feedback by $28.8 \%$ of the patients highlighted that being charged for the service was an additional burden on them.

There are several benefits of using telemedicine to deliver palliative care services to patients, especially during a pandemic where social interactions have to be reduced and manpower constraints are high. In the time of a "new normal", telemedicine will be increasingly employed to amplify the capacity of clinicians' healthcare networks. ${ }^{5}$ Our study has shown that the uptake of telemedicine by patients with advanced cancer in Singapore is associated with sociodemographic factors and that palliative care telemedicine services are acceptable.

Palliative care has traditionally been seen as a discipline that is high-touch rather than high-tech, ${ }^{6}$ and while it is possible for support of physical symptoms to be delivered via telemedicine, it can be difficult to provide psycho-emotional comfort and discuss end-of-life issues via telemedicine. ${ }^{7-10}$ This could offer an explanation as to why many patients still prefer in-person palliative care consultations over virtual consultations. To ensure that patients are not excluded from receiving palliative care due to low technological literacy, telemedicine applications and online questionnaires should be designed to be more userfriendly. Future efforts should also be tailored to promote the concept and feasibility of telemedicine in palliative care to the various sociodemographic groups accordingly, including consideration for appropriate use of financial reimbursements to increase uptake.

\section{Acknowledgement}

This study is funded by the SingHealth Duke-NUS Academic Medicine Special Request for Urgent COVID-19 Research Funding Grant (Reference Number: AM/CSP004/2020).

\section{REFERENCES}

1. Ahmed A, Siddiqi AR, Tahir MJ, et al. Use of telemedicine in healthcare during COVID-19 in Pakistan: Lessons, legislation challenges and future perspective. Ann Acad Med Singap 2021;50:485-6.

2. Ministry of Health. Continuation of Essential Healthcare Services During Period of Heightened Safe Distancing Measures, 4 April 2020. Available at: https://www.moh.gov.sg/news-highlights/details/ continuation-of-essential-healthcare-services-during-period-ofheightened-safe-distancing-measures. Accessed on 8 October 2021. 
3. Ho S, Tan YY, Neo SHS, et al. Covid 19 - A review of the impact it has made on supportive and palliative care services within a tertiary hospital and cancer centre in Singapore. Ann Acad Med Singap 2020;49:489-95.

4. Steindal SA, Nes AAG, Godskesen TE, et al. Patients' experience of telehealth in palliative care home care: scoping review. J Med Internet Res 2020;22:e16218.

5. Eastman O, Dowd A, White J, et al. Telehealth: rapid adoption in community palliative care due to COVID-19: patient and professional evaluation. BMJ Support Palliat Care 2021. doi: 10.1136/ bmjspcare-2021-002987.

6. Head BA, Schapmire T, Zheng Y. Telehealth in palliative care: A systematic review of patient-reported outcomes. J Hosp Palliat Nurs 2017;19:130-9.

7. Gorst SL, Armitage CJ, Brownsell S, et al. Home telehealth uptake and continued use among heart failure and chronic obstructive pulmonary disease patients: a systematic review. Ann Behav Med 2014;48:323-36

8. Rykkje L, Hjorth GHB. "Safety at home": Experiences from testing of video communication between patients and home health care personnel. SAGE Open 2017. doi: 10.1177/2158244017744900.
9. Calton B, Shibley WP, Cohen E, et al. Patient and caregiver experience with outpatient palliative care telemedicine visits. Palliat Med Rep 2020;1:339-46.

10. Chan RJ, Crichton M, Crawford-Williams F, et al. The efficacy, challenges and facilitators of telemedicine in post-treatment cancer survivorship care: an overview of systematic reviews. Ann Oncol 2021. doi: 10.1016/j.annonc.2021.09.001.

Kah Mun Natalie Mok ${ }^{1}{ }_{B S o c S c i}$ (Hons), Xia $\underline{\mathrm{Zhu}}{ }^{1} M N$, Xin Hui $\mathrm{Ng}^{1}{ }_{B N}$, Hui Shan Shirlyn Neo ${ }^{1}$ MRCP (Int Med)

${ }^{1}$ Division of Supportive and Palliative Care, National Cancer Centre Singapore

Correspondence: Dr Hui Shan Shirlyn Neo, Division of Supportive and Palliative Care, National Cancer Centre Singapore, 11 Hospital Crescent, Singapore 169610 .

Email: shirlyn.neo.h.s@singhealth.com.sg 